\title{
Identification of excitons, trions and biexcitons in single-layer $\mathrm{WS}_{2}$
}

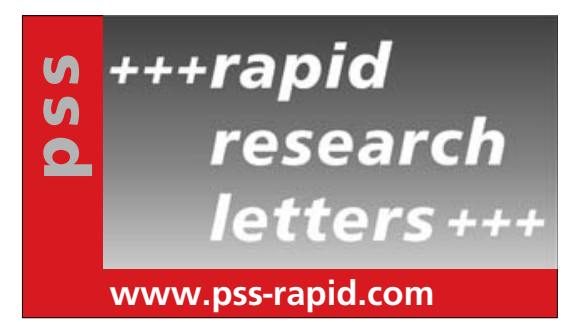

\section{Gerd Plechinger, Philipp Nagler, Julia Kraus, Nicola Paradiso, Christoph Strunk, Christian Schüller, and Tobias Korn}

Institut für Experimentelle und Angewandte Physik, Universität Regensburg, 93040 Regensburg, Germany

Received 8 July 2015, revised 22 July 2015, accepted 23 July 2015

Published online 27 July 2015

Keywords dichalcogenides, $\mathrm{WS}_{2}$, photoluminescence, excitons, trions, biexcitons, direct-gap semiconductors

* Corresponding author: e-mail tobias.korn@physik.uni-regensburg.de, Phone: +49-941-9432055, Fax: +49-941-9434226

Single-layer $\mathrm{WS}_{2}$ is a direct-gap semiconductor showing strong excitonic photoluminescence features in the visible spectral range. Here, we present temperature-dependent photoluminescence measurements on mechanically exfoliated single-layer $\mathrm{WS}_{2}$, revealing the existence of neutral and charged excitons at low temperatures as well as at room tem- perature. By applying a gate voltage, we can electrically control the ratio of excitons and trions and assert a residual ntype doping of our samples. At high excitation densities and low temperatures, an additional peak at energies below the trion dominates the photoluminescence, which we identify as biexciton emission.
1 Introduction In recent years, semiconducting, atomically thin transition metal dichalcogenides (TMDCs) like $\mathrm{MoS}_{2}, \mathrm{MoSe}_{2}, \mathrm{WSe}_{2}$ and $\mathrm{WS}_{2}$, have emerged as highly interesting materials for the scientific community due to their extraordinary optical [1] and electrical properties [2], including coupled spin-valley effects [3] and photovoltaic applications [4]. These molecular layers show strong photoluminescence (PL) peaks in the visible and near-infrared spectral range, as they experience a transition from an indirect gap in bulk and few-layer samples to a direct gap in the single-layer regime [5]. The spatial confinement of carriers in a two-dimensional layer and the weak dielectric screening lead to unusually strong excitonic effects $[6,7]$, even at room temperature. High exciton binding energies of the order of $0.5 \mathrm{eV}$ have been reported for single-layer $\mathrm{WS}_{2}$ [8-11]. Besides the charge-neutral exciton (X), i.e., a bound state of an electron and a hole, also charged excitons can be excited in the presence of residual excess charge carriers. These quasiparticles, called trions, consist either of two electrons and one hole $\left(\mathrm{X}^{-}\right)$or one electron and two holes $\left(\mathrm{X}^{+}\right)$. By applying a gate voltage, one can tune the spectral weight of charge-neutral excitons and trions in single-layer $\mathrm{MoS}_{2}$ [12], $\mathrm{MoSe}_{2}$ [13], $\mathrm{WS}_{2}$ [10] and $\mathrm{WSe}_{2}$ [14]. Additional, lower-energy PL emission peaks are observed in most single-layer TMDCs at low temperatures. These have been attributed to surface-adsorbate-bound excitons in $\mathrm{MoS}_{2}$ [15] and to crystal-defect-bound exciton states in single-layer diselenides [16, 17]. Given the large binding energy of the excitons, the formation of molecular states consisting of two excitons, so-called biexcitons [18], is to be expected in dichalcogenide single-layers. Biexciton PL emission should be at energies below the exciton emission due to the additional binding energy, in a similar energy range as defect-bound exciton emission. The signature of biexciton emission was recently observed in PL measurements on $\mathrm{WSe}_{2}$ [19]. Thus, the origin of the lowerenergy PL emission peaks in the other semiconducting TMDCs warrants close investigation.

In this work, we report on low-temperature PL of mechanically exfoliated single-layer $\mathrm{WS}_{2}$. To date, only a few works exist which report on the observation of excitons and trions in the low-temperature PL spectrum of mechanically exfoliated $[20,21]$ single-layer $\mathrm{WS}_{2}$. To the best of our knowledge, a thorough analysis of the temperaturedependent PL spectrum is still absent. In contrast to other semiconducting TMDCs, there is no consensus about the assignment of the $\mathrm{X}$ and $\mathrm{X}^{-} \mathrm{PL}$ features in low-temperature $\mathrm{PL}$ of single-layer $\mathrm{WS}_{2}$. The aim of this paper is to clarify those issues, and to provide insight into the nature of an additional low-energy peak in the PL spectrum, which is observable at low temperatures. We identify the exciton and the trion peaks in the temperature range from $295 \mathrm{~K}$ to $4 \mathrm{~K}$. Our interpretation of the PL spectra is substantiated by gate-dependent PL measurements which allow us to di- 
rectly control the exciton-trion ratio. Finally, we utilize power-dependent and helicity-resolved PL measurements to show that the low-energy PL peak we observe stems from a superposition of defect-bound exciton and biexciton emission.

2 Methods Our samples are mechanically exfoliated from bulk $\mathrm{WS}_{2}$ crystals (2d semiconductors inc.) onto a polydimethylsiloxane (PDMS) stamp. Using an optical microscope, we can identify single-layer flakes of $\mathrm{WS}_{2}$ on the PDMS stamp. We then transfer these flakes onto a p-doped Si chip with a $270 \mathrm{~nm} \mathrm{SiO}_{2}$ capping layer, applying an all-dry deterministic transfer procedure [22]. For gate-dependent measurements, we stamp the flakes onto p-doped Si chips with $500 \mathrm{~nm}$ thermal oxide and predefined metal contacts manufactured with e-beam lithography. We use the p-doped $\mathrm{Si}$ as a backgate. For lowtemperature measurements, the samples are mounted in a He-flow cryostat. The cw lasers used for excitation are focussed with a $100 \times$ microscope objective onto the sample, the emitted PL is collected by the same microscope objective (backscattering geometry) and guided into a spectrometer with a Peltier-cooled CCD chip. Unless otherwise noted, a $532 \mathrm{~nm}$ laser source is utilized. Helicityresolved measurements are performed using a $561 \mathrm{~nm}$ laser, which allows for near-resonant excitation. Further experimental details are published in Ref. [23].

3 Results and discussion Figure 1(a) shows the PL spectra of single-layer $\mathrm{WS}_{2}$ for various temperatures. In this measurement series, the laser excitation density is kept relatively low at $5 \mathrm{kWcm}^{-2}$ to avoid possible heating effects. At $295 \mathrm{~K}$, the spectrumconsists of two peaks at $2018 \mathrm{meV}$ and $1975 \mathrm{meV}$, which we attribute to the exciton $(\mathrm{X})$ and the trion $\left(\mathrm{X}^{-}\right)$. The peak positions at room temperature are in very good agreement with recent reports $[10,24,25]$. We note that even at room temperature, $X$ and $\mathrm{X}^{-}$peaks can be separated due to their small linewidth. The

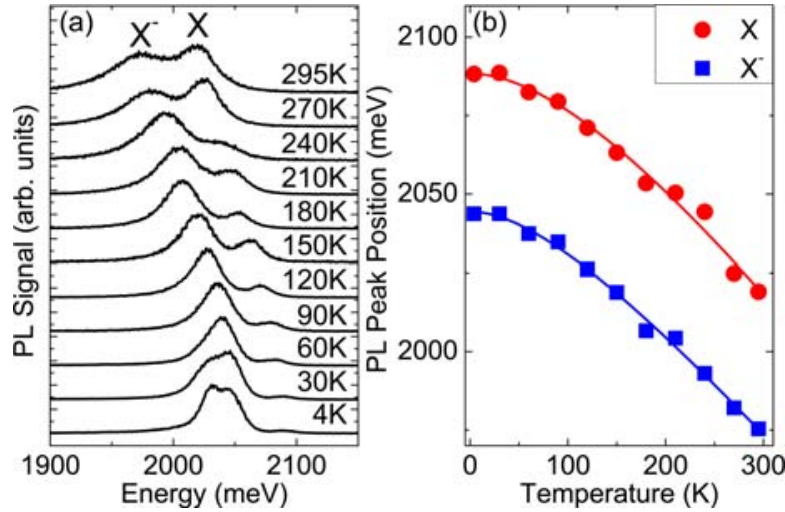

Figure 1 (a) Normalized PL spectra of single-layer $\mathrm{WS}_{2}$ for different temperatures. (b) Exciton (X) and trion $\left(\mathrm{X}^{-}\right) \mathrm{PL}$ peak energies as a function of temperature. The solid lines represent the fits to the experimental data following the Varshni equation. existence of the trion peak indicates an intrinsic doping of our sample, as it is commonly observed also in other TMDCs [2]. When cooling down the sample, both PL peaks experience a blueshift in accordance with the Varshni equation [26], which describes the change of the bandgap with temperature in a large variety of semiconductors,

$$
E_{\mathrm{g}}(T)=E_{\mathrm{g}}(0)-\alpha T^{2} /(T+\beta),
$$

where $E_{\mathrm{g}}(0)$ is the bandgap at zero temperature and $\alpha$ and $\beta$ are phenomenological fit parameters. We assume that the exciton and trion binding energy are temperature-independent, and that $\mathrm{X}$ and $\mathrm{X}^{-}$peaks rigidly shift with the bandgap. We use Eq. (1) to fit the PL peak positions extracted for each temperature, as depicted in Fig. 1(b). For both peaks, the fit matches with $\alpha=4.0 \times 10^{-4} \mathrm{eV} / \mathrm{K}$, $\beta=200 \mathrm{~K}$ and $E_{\mathrm{g}}(0)=2088 \mathrm{meV}$ for $\mathrm{X}$ and $E_{\mathrm{g}}(0)=$ $2045 \mathrm{meV}$ for $\mathrm{X}^{-}$. The parameters are comparable to those from previous studies on $\mathrm{MoS}_{2}$ [27]. Our assignment of the $\mathrm{X}$ and $\mathrm{X}^{-}$peaks at $T=4 \mathrm{~K}$ is further confirmed by additional power-dependent PL measurements at $150 \mathrm{~K}$ [28], in which we observe a low-energy tail in the $\mathrm{X}^{-}$peak, which is typical of an electron-recoil effect and has recently also been observed for trions in $\mathrm{MoSe}_{2}$ [13]. The spectral weight shifts from $\mathrm{X}$ to $\mathrm{X}^{-}$with decreasing temperature. This indicates that the thermal energy at higher temperatures is large enough to lead to a partial dissociation of the trions. In Fig. 1(a), we also see that the $\mathrm{X}^{-}$peak develops a low-energy shoulder at $30 \mathrm{~K}$ and, even more pronounced, at $4 \mathrm{~K}$, which we denominate as $\mathrm{L}_{1} / \mathrm{XX}$. We will demonstrate below that it stems from a superposition of defectbound exciton $\left(\mathrm{L}_{1}\right)$ and biexciton $(\mathrm{XX})$ emission. In previous reports, either the $\mathrm{L}_{1}$ and $\mathrm{L}_{2}$ peak [21] or the $\mathrm{X}^{-}$and $\mathrm{L}_{1}$ peak $[20,29]$, have been attributed to exciton and trion emission. The actual $\mathrm{X}$ peak at about $2.09 \mathrm{eV}$ is absent in those studies. The fact that we see a well-pronounced exciton peak in our spectra might be due to our sample preparation process, which leads to a reduced interaction with the substrate in comparison to direct exfoliation of flakes onto $\mathrm{SiO}_{2}$ using adhesive tape.

To confirm our assignment of the exciton and trion peaks, as well as the charge state of the trion, we perform gate-dependent PL measurements. The inset in Fig. 2(b) shows a microscope image of a gated sample. In Fig. 2(a), PL spectra are plotted for different backgate voltages $V_{\mathrm{g}}$ at room temperature. At large negative $V_{\mathrm{g}}$, the $\mathrm{X}$ peak is the dominant one, whereas it is completely suppressed for positive $V_{\mathrm{g}}$, where the $\mathrm{X}^{-}$peak is the only measurable feature. Hence, we infer that the trions in our samples are negatively charged. This indicates that the $\mathrm{WS}_{2}$ singlelayer has a residual n-type doping, similar to $\mathrm{MoS}_{2}$ [2] but in contrast to $\mathrm{WSe}_{2}$ [30]. Our room-temperature data is in perfect agreement with Ref. [10]. Figure 2(c) displays the gate-dependent PL spectra at $4 \mathrm{~K}$. For negative gate voltages, the $\mathrm{X}$ peak intensity increases as the Fermi level is shifted towards the neutral regime. This clearly confirms 

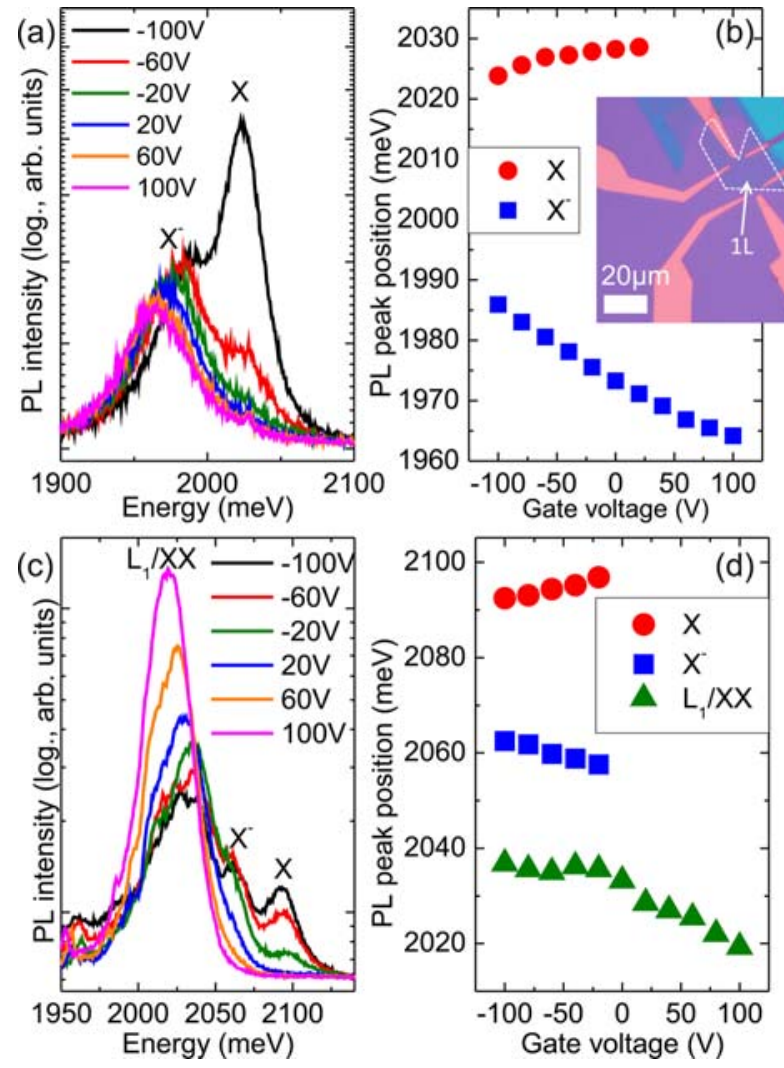

Figure 2 (a) PL spectra at room temperature for different gate voltages. (b) PL peak position of $\mathrm{X}$ and $\mathrm{X}^{-}$as a function of gate voltage. The inset shows an optical micrograph of the $\mathrm{WS}_{2}$ flake on a $\mathrm{Si} / \mathrm{SiO}_{2}$ substrate with prestructured $\mathrm{Ti}: \mathrm{Au}$ contacts. (c) PL spectra at $T=4 \mathrm{~K}$ for different gate voltages. (d) PL peak position of $\mathrm{X}, \mathrm{X}^{-}$and $\mathrm{L}_{1} / \mathrm{XX}$ peak as a function of gate voltage.

the identification of the $2.088 \mathrm{eV}$ peak as the exciton peak. The $\mathrm{X}^{-}$peak, in contrast, gains in intensity by increasing the gate voltage for $V_{\mathrm{g}}>0$. In both gate-voltage dependent measurement series, we observe that the $\mathrm{X}^{-}$peak experiences a spectral redshift, while the $X$ peak shows a slight blueshift with increasing $V_{\mathrm{g}}$ (Fig. 2(b) and (d)), so that the energy difference between $X$ and $\mathrm{X}^{-}$peaks increases with increasing carrier concentration. This effect has also been observed in other TMDCs $[12,13]$. In the limit of low carrier concentration, the ionization energy of a trion is equal to the trion binding energy.In the presence of a $2 \mathrm{D}$ electron gas (2DEG), however, ionization of a trion requires that the ionized electron is excited to a state above the Fermi energy of the 2DEG, as all states below the Fermi energy are occupied. Thus, the energy difference between exciton and trion peaks is given by [12]

$$
E_{\mathrm{X}}-E_{\mathrm{X}^{-}}=E_{\mathrm{b}, \mathrm{X}^{-}}+E_{\mathrm{F}},
$$

with $E_{\mathrm{X}}$ and $E_{\mathrm{X}^{-}}$being the exciton and trion PL peak energies, $E_{\mathrm{b}, \mathrm{X}^{-}}$the trion binding energy and $E_{\mathrm{F}}$ the Fermi energy, which is proportional to $V_{\mathrm{g}}$. Due to intrinsic doping and the corresponding non-zero $E_{\mathrm{F}}$, the measured exciton- trion energy difference $E_{\mathrm{X}}-E_{\mathrm{x}^{-}}$of $43 \mathrm{meV}$ in the ungated sample shown in Fig. 1(a) is larger than the actual trion binding energy. The exciton-trion peak separation in gated samples follows Eq. (2) [28], showing a minimal peak separation of $30 \mathrm{meV}$ at $V_{\mathrm{g}}=-100 \mathrm{~V}$. This represents an upper limit for the trion binding energy.

Finally, we focus on the low-energy feature labeled as $\mathrm{L}_{1} / \mathrm{XX}$ that arises at temperatures below $60 \mathrm{~K}$. Figure 3(a) shows the PL spectra at $T=4 \mathrm{~K}$ for different excitation powers. Whereas at low powers, $\mathrm{X}^{-}$and $\mathrm{L}_{1} / \mathrm{XX}$ are spectrally well separated and of similar intensity, at higher excitation powers, the $\mathrm{L}_{1} / \mathrm{XX}$ peak completely dominates the spectrum. Additionally, a second low-energy peak $\mathrm{L}_{2}$ with moderate intensity is discernible around $1.98 \mathrm{eV}$. It may stem from defect-bound excitons, as its intensity decreases relative to the other peaks with increasing excitation density. To get a better insight into the nature of the $\mathrm{L}_{1} / \mathrm{XX}$ peak, we extract the integrated PL intensity for $\mathrm{L}_{1} / \mathrm{XX}, \mathrm{X}^{-}$ and $\mathrm{X}$ for different excitation densities, as displayed in the double-logarithmic graph in Fig. 3(b). $X$ and $X^{-}$show a rather linear behavior indicated by the orange solid line, as expected for an excitonic feature [18]. In contrast, the $\mathrm{L}_{1} / \mathrm{XX}$ peak exhibits a linear dependence at low excitation density, while for excitation densities larger than $25 \mathrm{kWcm}^{-2}$, the data is well-described by a quadratic fit, indicated by the green solid line in Fig. 3(b). Such a quadratic increase in PL emission intensity is expected for biexcitons [31], although smaller, superlinear slopes are often observed in experiment due to the kinetics of biexciton formation and exciton recombination [19]. The different behavior for low and high excitation densities indicates that in fact, two different emission lines are responsible for the observed $\mathrm{L}_{1} / \mathrm{XX}$ peak: at low excitation density, the main contribution to the PL at the $\mathrm{L}_{1} / \mathrm{XX}$ peak position stems from defect-bound excitons (denominated $\mathrm{L}_{1}$ ). At high excitation density, the biexciton (XX) emission is dominant. To confirm our interpretation, we perform an excitationdensity dependent measurement series utilizing nearresonant, circularly-polarized excitation. Figure 3(c) shows helicity-resolved PL spectra measured at $4 \mathrm{~K}$ using different excitation densities. At low excitation density, $\mathrm{L}_{1} / \mathrm{XX}$, $\mathrm{X}^{-}$and $\mathrm{X}$ peaks are clearly observable, together with a spectrally broad feature at lower energy. This feature is reminiscent of low-temperature PL spectra of $\mathrm{MoS}_{2}$, where it is attributed to surface-adsorbate-bound excitons [15]. For this feature, co- and contra-circularly-polarized PL spectra have the same intensity, indicating no circular polarization. By contrast, $\mathrm{L}_{1} / \mathrm{XX}, \mathrm{X}^{-}$and $\mathrm{X}$ peaks show a clear intensity difference in the helicity-resolved PL. With increasing excitation density, the $\mathrm{L}_{1} / \mathrm{XX}$ emission begins to dominate the spectrum. For higher excitation densities, in the same range for which we observe the quadratic increase of the PL intensity discussed above, the $\mathrm{L}_{1} / \mathrm{XX}$ peak position shows a pronounced redshift and its polarization degree increases. These two observations are analyzed and compared to the behavior of the $\mathrm{X}^{-}$and $\mathrm{X}$ peaks in Fig. 3(d) and (e). The circular polarization degree of the 

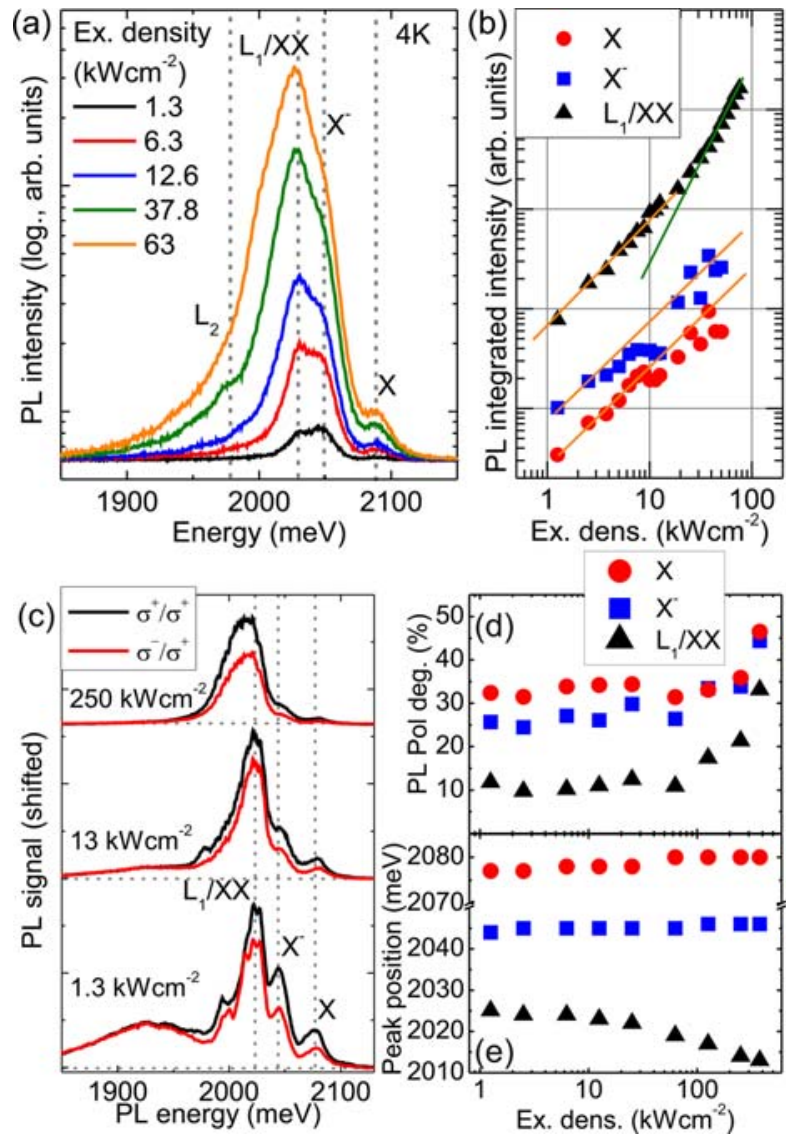

Figure 3 (a) PL spectra of single-layer $\mathrm{WS}_{2}$ at $T=4 \mathrm{~K}$ for various excitation densities. (b) Double-logarithmic plot of integrated $\mathrm{PL}$ intensity of $\mathrm{X}$ (red circles), $\mathrm{X}^{-}$(blue squares) and $\mathrm{L}_{1} / \mathrm{XX}$ peak (black triangles) as a function of excitation density. The orange solid lines indicate a linear dependency, whereas the green solid line indicates a quadratic dependency. (c) Helicity-resolved PL spectra of single-layer $\mathrm{WS}_{2}$ at $T=4 \mathrm{~K}$ under near-resonant, circularly-polarized excitation for various excitation densities. The black and red spectra are for co-circular and contra-circular excitation and detection, respectively. (d) PL circular polarization degree and (e) $\mathrm{PL}$ peak position for $\mathrm{X}, \mathrm{X}^{-}$and $\mathrm{L}_{1} / \mathrm{XX}$ peaks as a function of excitation density.

PL emission in single-layer TMDCs is an indicator of valley polarization, and for defect-related PL peaks, low values have been reported. By contrast, excitons, trions and biexcitons should show a significant PL polarization degree under near-resonant excitation [19]. In Fig. 3(d), we show that the PL polarization for the $\mathrm{X}^{-}$and $\mathrm{X}$ peaks is high and remains almost constant throughout the investigated excitation density range. By contrast, the $\mathrm{L}_{1} / \mathrm{XX}$ peak has a low PL polarization degree at low excitation density, indicative of defect-related PL emission. The PL polarization degree increases with increasing excitation density, as expected for biexciton emission, reaching similar values as the $\mathrm{X}^{-}$peak for the highest excitation density values in our series. As shown in Fig. 3(e), the $\mathrm{L}_{1} / \mathrm{XX}$ peak redshifts by about $10 \mathrm{meV}$ in the investigated excitation density range. This indicates that the $\mathrm{L}_{1}$ emission from de- fect-bound excitons at low excitation density is at a higher energy than the biexciton emission at high excitation density. We exclude local heating induced by the laser as a source of the redshift for the $\mathrm{L}_{1} / \mathrm{XX}$, since neither $\mathrm{X}^{-}$or $\mathrm{X}$ peaks display a redshift - by contrast, they show a slight blueshift. Thus, we can interpret the energy separation of about $65 \mathrm{meV}$ between the $\mathrm{X}$ and $\mathrm{XX}$ features as the biexciton binding energy $E_{\mathrm{b}, \mathrm{xx}}$. Currently, the value of the exciton binding energy in single-layer $\mathrm{WS}_{2}$ is still under discussion. The values determined in different experiments range between $320 \mathrm{meV} \mathrm{[8]} \mathrm{and} 700 \mathrm{meV}$ [9]. Thus, the Haynes factor, i.e., the ratio of $E_{\mathrm{b}, \mathrm{xx}}$ and the exciton binding energy, ranges between 9 and 20 percent, which is comparable to values for biexcitons in quantum wells [18] and those observed in $\mathrm{WSe}_{2}$ [19]. Remarkably, in our $\mathrm{WS}_{2}$ samples, strong biexciton PL emission is observable already under $\mathrm{cw}$ laser excitation, while pulsed excitation was required to study biexciton emission in $\mathrm{WSe}_{2}$ [19]. This indicates pronounced differences in the kinetics of biexciton formation in different TMDCs.

4 Conclusion In conclusion, we have presented temperature-dependent PL measurements on mechanically exfoliated single-layer $\mathrm{WS}_{2}$. We find that the exciton and trion peaks are well separated even in the room temperature spectrum and their emission can be tracked downto $4 \mathrm{~K}$. By tuning the Fermi level in our samples, we can unambiguously assign the $2.09 \mathrm{eV}$ PL peak to exciton and the $2.05 \mathrm{eV}$ PL peak to trion emission at $T=4 \mathrm{~K}$. At low temperatures, we observe the emergence of a lower-energy peak, which we identify as a superposition of defect-bound exciton and biexciton emission by the power dependence of its emission intensity and circular polarization degree. These results clarify some issues in the interpretation of low-temperature PL spectra in single-layer $\mathrm{WS}_{2}$, which is a promising candidate for all-2D electrooptical and valleytronic devices.

Acknowledgements The authors ackowledge financial support by the DFG via KO3612/1-1, GRK1570 and SFB689.

Supporting Information Additional supporting information may be found in the online version of this article at the publisher's website.

\section{References}

[1] K. F. Mak, C. Lee, J. Hone, J. Shan, and T. F. Heinz, Phys. Rev. Lett. 105, 136805 (2010).

[2] B. Radisavljevic, A. Radenovic, J. Brivio, V. Giacometti, and A. Kis, Nature Nanotechnol. 6, 147-150 (2011).

[3] K. F. Mak, K. He, J. Shan, and T. F. Heinz, Nature Nanotechnol. 7, 494-498 (2012).

[4] M. M. Furchi, A. Pospischil, F. Libisch, J. Burgdörfer, and T. Mueller, Nano Lett. 14, 4785-4791 (2014).

[5] A. Splendiani, L. Sun, Y. Zhang, T. Li, J. Kim, C. Y. Chim, G. Galli, and F. Wang, Nano Lett. 10, 1271-1275 (2010).

[6] T. Cheiwchanchamnangij and W. R. L. Lambrecht, Phys. Rev. B 85, 205302 (2012). 
[7] G. Berghäuser and E. Malic, Phys. Rev. B 89, 125309 (2014).

[8] A. Chernikov, T. C. Berkelbach, H. M. Hill, A. Rigosi, Y. Li, O. B. Aslan, D. R. Reichman, M. S. Hybertsen, and T. F. Heinz, Phys. Rev. Lett. 113, 076802 (2014).

[9] Z. Ye, T. Cao, K. O’Brien, H. Zhu, X. Yin, Y. Wang, S. G. Louie, and X. Zhang, Nature 513, 214-218 (2014).

[10] B. Zhu, X. Chen, and X. Cui, Sci. Rep. 5, 9218 (2015).

[11] A. T. Hanbicki, M. Currie, G. Kioseoglou, A. L. Friedman, and B. T. Jonker, Solid State Commun. 203, 16-20 (2015).

[12] K. F. Mak, K. He, C. Lee, G. H. Lee, J. Hone, T. F. Heinz, and J. Shan, Nature Mater. 12, 207-211 (2013).

[13] J. S. Ross, S. F. Wu, H. Y. Yu, N. J. Ghimire, A. M. Jones, G. Aivazian, J. Q. Yan, D. G. Mandrus, D. Xiao, W. Yao, and X. Xu, Nature Commun. 4, 1474 (2013).

[14] A. M. Jones, H. Yu, N. J. Ghimire, S. Wu, G. Aivazian, J. S. Ross, B. Zhao, J. Yan, D. G. Mandrus, D. Xiao, W. Yao, and X. Xu, Nature Nanotechnol. 8, 634-638 (2013).

[15] G. Plechinger, F. X. Schrettenbrunner, J. Eroms, D. Weiss, C. Schüller, and T. Korn, Phys. Status Solidi RRL 6, 126128 (2012).

[16] G. Wang, L. Bouet, D. Lagarde, M. Vidal, A. Balocchi, T. Amand, X. Marie, and B. Urbaszek, Phys. Rev. B 90, 075413 (2014).

[17] G. Wang, L. Bouet, D. Lagarde, M. Vidal, A. Balocchi, T. Amand, X. Marie, and B. Urbaszek, Phys. Rev. B 90, 075413 (2014).

[18] C. F. Klingshirn (ed.), Semiconductor Optics (Springer, 2007).

[19] Y. You, X. X. Zhang, T. C. Berkelbach, M. S. Hybertsen, D. R. Reichman, and T. F. Heinz, Nature Phys. 11, 477-481 (2015).
[20] T. Scrace, Y. Tsai, B. Barman, L. Scheidenback, A. Petrou, G. Kioseoglou, I. Ozfidan, M. Korkusinski, and P. Hawrylak, Nature Nanotechnol. (2015), 2015/05/11/online.

[21] A. A. Mitioglu, P. Plochocka, J. N. Jadczak, W. Escoffier, G. L. J. A. Rikken, L. Kulyuk, and D. K. Maude, Phys. Rev. B 88, 245403 (2013).

[22] A. Castellanos-Gomez, M. Buscema, R. Molendaar, V. Singh, L. Janssen, H. S. J. van der Zant, and G. A. Steele, 2D Materials 1, 011002 (2014).

[23] G. Plechinger, J. Mann, E. Preciado, D. Barroso, A. Nguyen, J. Eroms, C. Schüller, L. Bartels, and T. Korn, Semicond. Sci. Technol. 29, 064008 (2014).

[24] N. Peimyoo, W. Yang, J. Shang, X. Shen, Y. Wang, and T. Yu, ACS Nano 8, 11320-11329 (2014).

[25] M. Z. Bellus, F. Ceballos, H. Y. Chiu, and H. Zhao, ACS Nano 9(6), 6459-6464 (2015).

[26] Y. Varshni, Physica 34, 149 (1967).

[27] T. Korn, S. Heydrich, M. Hirmer, J. Schmutzler, and C. Schüller, Appl. Phys. Lett. 99, 102109 (2011).

[28] See Supplementary Information for further details.

[29] Y. Ye, Z. J. Wong, X. Lu, H. Zhu, X. Chen, Y. Wang, and X. Zhang, arXiv:1503.06141 (2015).

[30] C. H. Lee, G. H. Lee, A. M. van der Zande, W. Chen, Y. Li, M. Han, X. Cui, G. Arefe, C. Nuckolls, T. F. Heinz, J. Guo, J. Hone, and P. Kim, Nature Nanotechnol. 9, 676-681 (2014).

[31] P. L. Gourley and J. P. Wolfe, Phys. Rev. B 20, 3319-3327 (1979). 